\title{
External disturbance rejection in IDA-PBC controller for underactuated mechanical systems : from theory to real time experiments
}

\author{
N.Khraief Haddad ${ }^{1}$, A.Chemori ${ }^{2}$ and S.Belghith ${ }^{3}$
}

\begin{abstract}
Proving the robustness, vis-à-vis external disturbances of IDA-PBC (Interconnexion Damping Assignment, Passive Based Control) controller for underactuated mechanical systems is addressed in this paper. Some sufficient stability conditions on matched and unmatched disturbances are provided. As illustration we propose to revisit the application of IDA-PBC controller to the Inertia Wheel Inverted Pendulum (IWIP) in the presence of external disturbances. Simulations and real-time experimental results are presented as validations of the theoretical results.
\end{abstract}

\section{INTRODUCTION}

In this paper we will consider the robustness analysis of IDA-PBC controller when applied to underactuated mechanical systems. The latter, will be, necessarily ${ }^{1}[1]$, described by the so-called Port Controlled Hamiltonian models (PCH). PCH systems have been introduced in 1994 by Van der Shaft and Mashke [2]. The central paradigm of complex systems modeling is to have individual open subsystems with well defined port interfaces, hiding an internal model of variable complexity, and a set of rules describing how these subsystems interact through the port variables [3]. To implement this general idea we can use the so called port Hamiltonian systems or port-controlled Hamiltonian systems (PCHS). PCH models represent another alternative to the classical Euler-Lagrange (or standard Hamiltonian) models. Writing a system in a PCH form has the advantage to cover a large set of physical systems and provide important structural properties. An extended survey of PCHS is presented in [4]. One procedure to control PCH systems is called IDAPBC [5]. It combines the passivity properties of PCHS with control by interconnection and energy based control. IDA$\mathrm{PBC}$ uses the hamiltonian framework, it consists in solving the PDE associated to the energy balance equation. This technique has been applied to various plants : Mechanical systems [6], [7], magnetic levitation systems [8], [9], mass balance systems [10], electrical machines [11], [12], power converters [13]. For an in-depth review of IDA-PBC the reader is referred to [14].

In this paper we will study the robustness of IDA-PBC, for underactuated mechanical systems. We will focus on the

\footnotetext{
${ }^{1}$ National School of Engineers of Tunis, University of Tunis El Manar, BP.37, le Belvédère, 1002 Tunis, Tunisia (khraiefnahla@gmail.com).

${ }^{2}$ LIRMM, University of Montpellier 2, CNRS, 161 rue Ada, 34392 Montpellier, France (Ahmed. Chemori@lirmm.fr)

${ }^{3}$ National School of Engineers of Tunis, University of Tunis El Manar, BP.37, le Belvédère, 1002 Tunis, Tunisia (safya.belghith@enit.rnu.tn).

1. To stabilize underactuated mechanical systems by energy shaping, it is necessary to modify the total energy function. Which cannot be done with the classical PBC (passivity based control). That is why IDA-PBC is used.
}

case of external disturbances where little is known. Many studies turn around the improvement of the robustness. Input disturbance suppression for PCHS using an internal model, has been studied in [15]. In [9] an IDA-PBC controller applied to a magnet levitation system was experimentally tested. To solve the robustness problem they add an integral term to the error of the passive output. The same technique was used in [16] to improve the robustness of hamiltonian passive control. Stability and robustness of disturbed portcontrolled Hamiltonian systems with dissipation have been addressed in [17]. The authors studied IDA-PBC controller robustness against parameters uncertainties. Recently Jose Guadalupe Romero et al. [18] improve the robustness vis$\grave{a}$-vis external disturbance, of energy shaping controllers for fully actuated mechanical systems. They design a dynamic state feedback controller such that the closed-loop system ensures some stability properties in spite of the presence of external disturbances. Nevertheless two important issues are still open :

1) The proof of the robustness of the controller obtained by IDA-PBC technique.

2) More real-time experimental applications when applying IDA-PBC controller.

Motivated by both issues and as a main contribution of this paper, we prove that, when applying IDA-PBC to underactuated mechanical systems, the stability is preserved in spite of the presence of external disturbances. Some sufficient stability conditions on the external disturbances are given. To strength those results we propose to validate the proposed controller experimentally on the well known inertia wheel inverted pendulum (IWIP). Based on the IDA-PBC controller designed in [6], we did some experiments in presence of external disturbances. The robustness of IDA-PBC controller is proved through these experimental results.

The remaining of the paper is organized as follows. In section II, background on IDA-PBC for underactuated systems is given. Section III introduces the main contribution about external disturbance rejection in IDA-PBC controllers. Simulations and experimental results on an inertia wheel inverted pendulum are given in section IV. Finally, we present some conclusions and future work in section V.

\section{BACKGROUND ON IDA-PBC CONTROL}

In this section let's consider previous work proposed in [6], [5]. An underactuated mechanical system whith no natural 
damping can be written in $\mathrm{PCH}$ form as follows ${ }^{2}$ :

$$
\left\{\begin{aligned}
\left(\begin{array}{c}
\dot{q} \\
\dot{p}
\end{array}\right) & =\left(\begin{array}{cc}
0 & I_{n} \\
-I_{n} & 0
\end{array}\right)\left(\begin{array}{c}
\nabla_{q} H \\
\nabla_{p} H
\end{array}\right)+\left(\begin{array}{c}
0 \\
G(q)
\end{array}\right) u \\
y & =G(q)^{T} \nabla_{p} H
\end{aligned}\right.
$$

with total energy

$$
H(q, p)=\frac{1}{2} p^{T} M^{-1}(q) p+V(q)
$$

(1) will be called nominal system, where $q \in \mathbb{R}^{n}, p \in \mathbb{R}^{n}$ are the generalized position and momenta respectively. $G(q) \in$ $\mathbb{R}^{n \times m}$, is the input matrix. We consider here that the system is underactuated and assume $\operatorname{rank}(G)=m<n, u$ and $y$ are the control input vector and the output vector respectively. $M(q)=M^{T}(q)>0$ is the inertia matrix, and $V(q)$ is the potential energy. We have that $\dot{q}=M^{-1}(q) p$.

The desired (closed loop) enregy function is given by :

$$
H_{d}(q, p)=\frac{1}{2} p^{T} M_{d}^{-1}(q) p+V_{d}(q)
$$

we define $\left(q^{*}, 0\right)$ as the desired equilibrium. $V_{d}$ is required to have an isolated minimum at $q^{*}$. This target is achieved by the following IDA-PBC controller [6] :

$u=\underbrace{\left(G^{T} G\right)^{-1} G^{T}\left(\nabla_{q} H-M_{d} M^{-1} \nabla_{q} H_{d}+J_{2} M_{d}^{-1} p\right)}_{u_{e s}}+\underbrace{\left(-K_{v}\right) G^{T} \nabla_{p} H_{d}}_{u_{d i}}$

$u_{e s}$ is the energy shaping control to assign the equilibrium and $u_{d i}$ injects damping to achieve asymptotic stability. We obtain finally, the following desired (closed loop) PCH dynamics :

$$
\left\{\begin{aligned}
\left(\begin{array}{c}
\dot{q} \\
\dot{p}
\end{array}\right) & =\left(J_{d}(q, p)-R_{d}(q, p)\right)\left(\begin{array}{c}
\nabla_{q} H_{d} \\
\nabla_{p} H_{d}
\end{array}\right) \\
y & =G(q)^{T} \nabla_{p} H_{d}
\end{aligned}\right.
$$

where $M_{d}=M_{d}^{T}>0, J_{d}=-J_{d}^{T}=\left(\begin{array}{cc}0 & M^{-1} M_{d} \\ -M_{d} M^{-1} & J_{2}\end{array}\right)$ is the interconnection matrix, and $R_{d}=R_{d}^{T}=\left(\begin{array}{cc}0 & 0 \\ 0 & G K_{v} G^{T}\end{array}\right)$ is the damping matrix [5].

\section{IDA-PBC FOR DISTURBED UNDERACTUATED MECHANICAL SYSTEMS}

\section{A. Problem formulation}

Describe disturbed underactuated mechanical system by the following $\mathrm{PCH}$ model :

$$
\left\{\begin{aligned}
\left(\begin{array}{l}
\dot{q} \\
\dot{p}
\end{array}\right) & =\left(\begin{array}{cc}
0 & I_{n} \\
-I_{n} & 0
\end{array}\right)\left(\begin{array}{c}
\nabla_{q} H \\
\nabla_{p} H
\end{array}\right)+\left(\begin{array}{c}
0 \\
G(q)
\end{array}\right)\left(u+\delta_{1}(x, t)\right)+\delta_{2}(x, t) \\
y & =G(q)^{T} \nabla_{p} H
\end{aligned}\right.
$$

where $t \in \mathbb{R}, x=\left(\begin{array}{ll}q & p\end{array}\right)^{T} \in \mathbb{R}^{2 n}$ is the state, $u(t) \in \mathbb{R}^{m}$ is the control, $\delta_{1}(x, t)$ is the matched uncertainties, and $\delta_{2}(x, t)$ is the unmatched uncertainties. Both $\delta_{1}(x, t)$ and $\delta_{2}(x, t)$ are assumed to be unmeasured and bounded in magnitude, usually their Euclidean norm is denoted by $\|$.$\| . In this$ paper we formulate our IDA-PBC stabilization objective as follows : Given the disturbed PCH system (6) and a desired

2. Through the whole of the paper we present all vectors, including the gradient, as column vectors. equilibrium $\left(q^{*}, 0\right)$, is the IDA-PBC controller (4) capable to reject disturbances and keep $\left(q^{*}, 0\right)$ always asymptotically stable ?. In particular we treat two cases :

- Preserving asymptotic stability in spite of the existence of matched disturbances.

- Preserving asymptotic stability in spite of the existence of unmatched disturbances.

\section{B. Main result}

Case $1:$ Matched uncertainties $\left(\delta_{2}(x, t)=0\right)$

Applying the controller (4) to (6) we obtain the following closed loop disturbed system :

$$
\left\{\begin{aligned}
\left(\begin{array}{c}
\dot{q} \\
\dot{p}
\end{array}\right) & =\left(J_{d}(q, p)-R_{d}(q, p)\right)\left(\begin{array}{c}
\nabla_{q} H_{d} \\
\nabla_{p} H_{d}
\end{array}\right)+\delta_{1}(x, t)^{\prime} \\
y & =G(q)^{T} \nabla_{p} H_{d}
\end{aligned}\right.
$$

Note that $\delta_{1}(x, t)^{\prime}=\left(\begin{array}{ll}0 & G(q) \delta_{1}(x, t)\end{array}\right)^{T}$ is also a vector of external disturbances. Let $\lambda_{\min }\left\{K_{v}\right\}$ the smallest eigenvalue of the matrix $K_{v}$, and $\widetilde{x}=\left(q-q^{*} p\right)^{T}$.

The following proposition set some sufficient conditions on the disturbances boundaries in order to get $\left(q^{*}, 0\right)$ asymptotically stable.

Proposition 1 :

Consider the closed loop dynamics (7) with the desired total energy $H_{d}$.

If $\left\|\delta_{1}(x, t)\right\| \leq \lambda_{\min }\left\{K_{v}\right\}\left|\left(\nabla_{p} H_{d}\right)^{T} G\right|^{2}$, then $\dot{H}_{d} \leq 0$ and $\widetilde{x}$ of (10) is an asymptotically stable equilibrium point. Proof:

$$
\begin{aligned}
& \begin{array}{l}
\dot{H}_{d}=\nabla_{q} H_{d} \dot{q}+\nabla_{p} H_{d} \dot{p} \\
=\left(\nabla_{q} H_{d}\right)^{T}\left(M^{-1} M_{d}\right) \nabla_{p} H_{d}+\left(\nabla_{p} H_{d}\right)^{T} G(q) \delta_{1}(x, t)- \\
\quad\left(\nabla_{p} H_{d}\right)^{T}\left(M_{d} M^{-1}\right) \nabla_{q} H_{d}+ \\
\quad\left(\nabla_{p} H_{d}\right)^{T}\left(J_{2}-G K_{v} G^{T}\right) \nabla_{p} H_{d} \\
=\left(\nabla_{p} H_{d}\right)^{T} G(q) \delta_{1}(x, t)-\left(\nabla_{p} H_{d}\right)^{T}\left(G K_{v} G^{T}\right) \nabla_{p} H_{d}
\end{array}
\end{aligned}
$$

If $\left\|\delta_{1}(x, t)\right\| \leq \lambda_{\min }\left\{K_{v}\right\}\left|\left(\nabla_{p} H_{d}\right)^{T} G\right|^{2}$ then

$$
\dot{H}_{d} \leq\left\|\delta_{1}(x, t)\right\|-\lambda_{\min }\left\{K_{v}\right\}\left|\left(\nabla_{p} H_{d}\right)^{T} G\right|^{2}
$$

and $\dot{H}_{d} \leq 0$

Case 2 : Unmatched uncertainties $\left(\delta_{1}(x, t)=0\right)$

Applying the controller (4) to (6) we obtain the following (69losed loop disturbed system :

$$
\left\{\begin{aligned}
\left(\begin{array}{c}
\dot{q} \\
\dot{p}
\end{array}\right) & =\left(J_{d}(q, p)-R_{d}(q, p)\right)\left(\begin{array}{c}
\nabla_{q} H_{d} \\
\nabla_{p} H_{d}
\end{array}\right)+\delta_{2}(x, t)^{\prime} \\
y & =G(q)^{T} \nabla_{p} H_{d}
\end{aligned}\right.
$$

Note that $\delta_{2}(x, t)^{\prime}=\delta_{2}(x, t)=\left(\delta_{21}(x, t) \quad \delta_{22}(x, t)\right)^{T}$, let $\lambda_{\min }\left\{K_{v}\right\}$ the smallest eigenvalue of the matrix $K_{v}$, and $\widetilde{x}=\left(\begin{array}{ll}q-q^{*} & p\end{array}\right)^{T}$.

The following proposition set some sufficient conditions on the disturbances boundaries in order to get $\left(q^{*}, 0\right)$ asymptotically stable.

Proposition2 : 
Consider the closed loop dynamics (10) with the desired total energy $H_{d}$. If $\left|\nabla_{x}^{T} H_{d} . \delta_{2}(x, t)\right| \leq \lambda_{\min }\left\{K_{v}\right\}\left|\left(\nabla_{p} H_{d}\right)^{T} G\right|^{2}$ then $\dot{H}_{d} \leq 0$ and $\widetilde{x}$ of (10) is an asymptotically stable equilibrium point. The proof of the proposition 2 is completed proceeding as in the case 1 .

\section{APPLICATION : INERTIA WHEEL INVERTED PENDULUM (IWIP)}

\section{A. IWIP : Description and modeling}

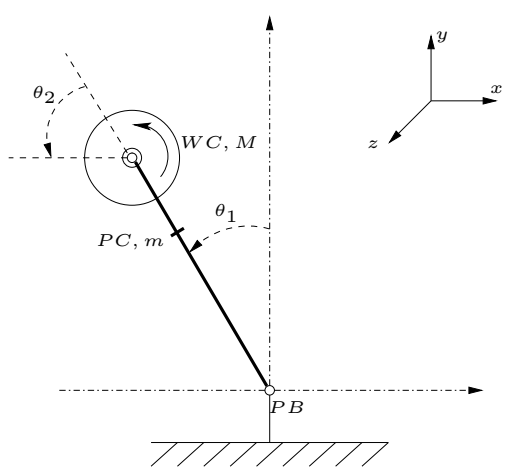

Fig. 1: Inertia Wheel Inverted Pendulum.

The pendulum device shown in Fig. 1 is called the Inertia Wheel Inverted Pendulum (IWIP). It may be modeled as a two-degrees-of-freedom serial mechanism. The first link is the pendulum and the second one is the rotating disc. The IWIP is an underactuated system, the torque produces an angular acceleration of the end-mass which generates a coupling torque at the pendulum axes. The parameters of the (IWIP) are shown in TABLE I :

\begin{tabular}{|l||c||c|}
\hline Description & Notation & Units \\
\hline \hline Pendulum angle with respect to vertical axis & $\theta_{1}$ & $\mathrm{rad}$ \\
\hline Wheel angle with respect to pendulum axis & $\theta_{2}$ & $\mathrm{rad}$ \\
\hline Mass of the pendulum & $\mathrm{m}$ & $\mathrm{kg}$ \\
\hline Mass of the Wheel & $\mathrm{M}$ & $\mathrm{kg}$ \\
\hline $\begin{array}{l}\text { Length from pendulum base (PB) to pendu- } \\
\text { lum center of mass (PC) }\end{array}$ & 1 & $\mathrm{~m}$ \\
\hline $\begin{array}{l}\text { Length from pendulum base (PB) to Wheel } \\
\text { center of mass (WC) }\end{array}$ & $\mathrm{L}$ & $\mathrm{m}$ \\
\hline $\begin{array}{l}\text { Rotational Inertia of pendulum about pen- } \\
\text { dulum center of mass (PC) }\end{array}$ & $I_{P C}$ & $\mathrm{~kg} \cdot \mathrm{m}^{2}$ \\
\hline $\begin{array}{l}\text { Rotational Inertia of pendulum about pen- } \\
\text { dulum base (PB) }\end{array}$ & $I_{P B}$ & $\mathrm{~kg} \cdot \mathrm{m}^{2}$ \\
\hline $\begin{array}{l}\text { Rotational Inertia of Wheel about Wheel } \\
\text { center of mass (WC) }\end{array}$ & $I_{W C}$ & $\mathrm{~m} / \mathrm{s}^{2}$ \\
\hline Constant of gravitational acceleration & $g$ & $\mathrm{~m}$ \\
\hline
\end{tabular}

TABLE I: Description of dynamical parameters of the IWIP

The Euler-Lagrange equations of motion can be written as
[19] :

$$
\left(\begin{array}{cc}
\left(a+I_{W C}\right) & I_{W C} \\
I_{W C} & I_{W C}
\end{array}\right)\left(\begin{array}{c}
\ddot{\theta_{1}} \\
\ddot{\theta_{2}}
\end{array}\right)-\left(\begin{array}{c}
b g \sin \theta_{1} \\
0
\end{array}\right)=\left(\begin{array}{l}
0 \\
u
\end{array}\right)
$$

where : $\theta=\left[\begin{array}{ll}\theta_{1} & \theta_{2}\end{array}\right]^{T}, u$ is the torque applied by the motor to spin the wheel and :

$$
\begin{aligned}
a & =M L^{2}+I_{P B} \\
b & =m l+M L
\end{aligned}
$$

Let's introduce the following change of coordinates :

$$
\left[\begin{array}{l}
q_{1} \\
q_{2}
\end{array}\right]=\left[\begin{array}{ll}
1 & 0 \\
1 & 1
\end{array}\right]\left[\begin{array}{l}
\theta_{1} \\
\theta_{2}
\end{array}\right]
$$

This leads to a simplified model :

$$
\left[\begin{array}{cc}
a & 0 \\
0 & I_{W C}
\end{array}\right]\left[\begin{array}{l}
\ddot{q}_{1} \\
\ddot{q} 2
\end{array}\right]-\left[\begin{array}{c}
b g \sin q_{1} \\
0
\end{array}\right]=\left[\begin{array}{c}
-1 \\
1
\end{array}\right] u .
$$

The model (14) can be written through Hamilton's equations of motion as [20] :

$$
\left[\begin{array}{c}
\dot{q_{1}} \\
\dot{q_{2}} \\
\dot{p_{1}} \\
\dot{p_{2}}
\end{array}\right]=\left[\begin{array}{c}
\frac{p_{1}}{a} \\
\frac{p_{2}}{I_{W C}} \\
b g \sin q_{1}-u \\
u
\end{array}\right]
$$

Where $q=\left[\begin{array}{ll}q_{1} & q_{2}\end{array}\right]^{T}$ and $p=\left[\begin{array}{ll}p_{1} & p_{2}\end{array}\right]^{T}=\left[\begin{array}{ll}a \dot{q_{1}} & I_{W C} \dot{q_{2}}\end{array}\right]^{T}$ are the generalized positions and momenta respectively. Whitch leads to write the Hamiltonian function :

$$
H(q, p)=\frac{1}{2} p^{T} M^{-1}(q) p=\frac{1}{2}\left[\frac{p_{1}^{2}}{a}+\frac{p_{2}^{2}}{I_{W C}}\right]
$$

At first we aim at applying IDA-PBC controller in order to globally stabilize the system around its unstable equilibrium (the upward position of the pendulum) with the inertia disk aligned, this equilibrium corresponds to $q_{1}^{*}=q_{2}^{*}=0$.

Then the desired Hamiltonian function can be defined as :

$$
H_{d}(q, p)=\frac{1}{2} p^{T} M_{d}^{-1}(q) p+V_{d}(q)
$$

where ${ }^{3} M_{d}=\left(\begin{array}{ll}a_{1} & a_{2} \\ a_{2} & a_{3}\end{array}\right)$ with $a_{1}>0, a_{1} a_{3}>a_{2}^{2}$ and $a_{1}+$ $a_{2}<0$. Secondly, the robustness of such controller will be tested in the presence of external, matched and unmatched, disturbances.

3. Note that the inertia matrix $M$ of the IWIP is independent of $q$, so we can take $J_{2}=0$ and $M_{d}$ a constant matrix [6] 


\begin{tabular}{|l|c||c|c|}
\hline Parameter & Description & Value & Unit \\
\hline$m$ & pendulum mass & 3.228 & $\mathrm{Kg}$ \\
$M$ & mass of the wheel & 0.33081 & $\mathrm{Kg}$ \\
$l$ & Pendulum center of mass position & 0.06 & $\mathrm{~m}$ \\
$L$ & Wheel center of mass position & 0.044 & $\mathrm{~m}$ \\
$I_{1}$ & Pendulum inertia & 0.0314 & $\mathrm{Kgm}^{2}$ \\
$I_{2}$ & Wheel inertia & $4.176 e-4$ & $\mathrm{Kgm}^{2}$ \\
\hline
\end{tabular}

TABLE II: Dynamic Parameters of the IWIP

\section{B. IDA-PBC controller}

In [6] the authors proposed the following control law for the IWIP :

$$
u=\gamma_{1} \sin \left(q_{1}\right)+k_{p}\left(q_{2}+\gamma_{2} q_{1}\right)+k_{v} k_{2}\left(\dot{q}_{2}+\gamma_{2} \dot{q}_{1}\right)
$$

where $\gamma_{1}>b, \gamma_{2}=-\frac{a \gamma_{1}}{I_{W} C\left(\gamma_{1}-b\right)}$ and $k_{2}>0$.

Together with $k_{p}$ and $k_{v}$ positive arbitrary constants, define the tuning gains.

In this paper we propose to rewrite the control law (18) in terms of the generalized coordinates $q$ and momenta $p$ as :

$$
u=\underbrace{\gamma_{1} \sin \left(q_{1}\right)+k_{1} q_{1}+k_{2} q_{2}}_{u_{e s}}+\underbrace{k_{3} p_{1}+k_{4} p_{2}}_{u_{d i}}
$$

where $: k_{1}=k_{p} \gamma_{2}, k_{2}=k_{p}, k_{3}=k_{v}\left(\frac{a_{2}+a_{3}}{a_{1} a_{3}-a_{2}^{2}}\right)$ and $k_{4}=$ $-k_{v}\left(\frac{a_{1}+a_{2}}{a_{1} a_{3}-a_{2}^{2}}\right)$.

\section{Simulation results}

We will consider in simulations the dynamics of the $\mathrm{PCH}$ model (15). The parameters of the IWIP are summarized in TABLE II. They have been obtained from the CAD of the system.

1) Stabilization with external disturbances: To test IDAPBC controller robustness for the IWIP, we will check in this section, its capability to reject external, matched and unmatched, disturbances.

Case 1 : Matched disturbances:

In this case a matched disturbance is added to the dynamics (15). It consists of constant torques $\left\|\delta_{1}(x, t)\right\|=10^{4}$ added to the control (19) at time instants : $t=5 s, t=10 \mathrm{~s}$ and $t=15 s$ during $0.2 s$ at each time. The obtained simulation results are shown in Fig. 2 and prove that the IDA-PBC controller is capable to compensate matched disturbances and keep the system around the open loop unstable equilibrium point. Case 2 : Unmatched disturbances :

In this case a vector of constant disturbances $\left\|\delta_{2}(x, t)\right\|=$ $[0.3 ;-0.315]^{5}$ is added to the dynamics of the IWIP at

4. we can check simply that $\left\|\delta_{1}(x, t)\right\| \leq 12 * \|\left|\left(\nabla_{p} H_{d}\right)^{T} G\right|^{2}$, leading the theoretical results.

5. we can check simply that $\left|\nabla_{x}^{T} H_{d} \cdot \delta_{2}(x, t)\right| \leq 12 * \|\left.\left(\nabla_{p} H_{d}\right)^{T} G\right|^{2}$, leading the theoretical results.
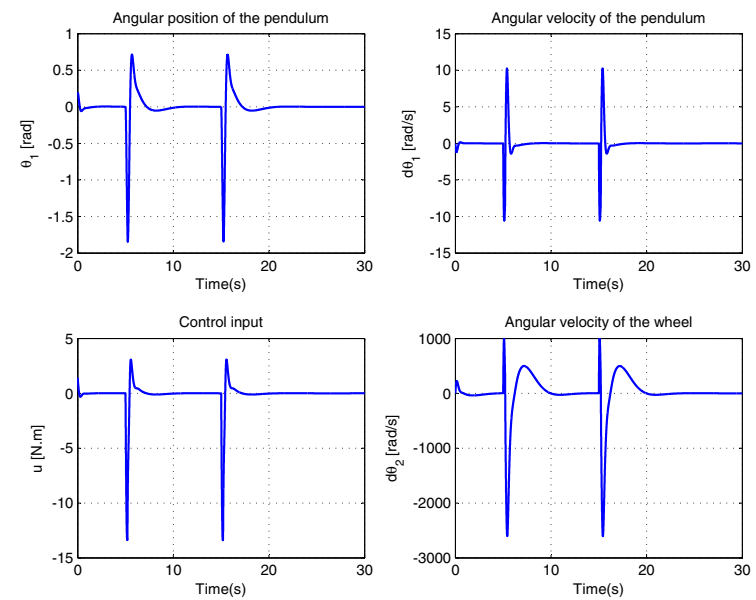

Fig. 2: Simulation results with matched disturbances

$t=10 s$. Fig. 3 displays the performance of IDA-PBC controller.

Note that we can check the disturbance boundary by varying the amplitude of the added vector of disturbances and we can observe the lost of stability of the system when exceeding a limit value (determining in section III) of the amplitude disturbance.
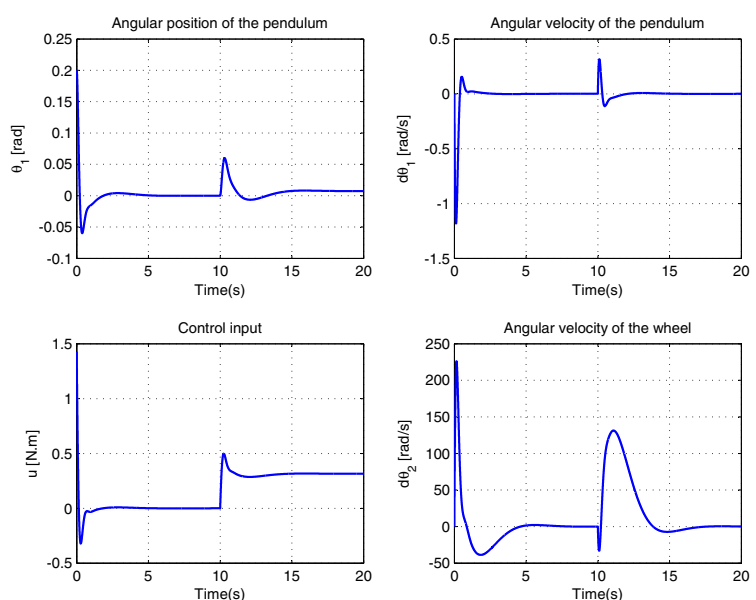

Fig. 3: Simulation results with unmatched disturbances D. Real-time experimental results

1) Experimental testbed: . To validate the theoretical results, real-time experiments are carried out on the inertia wheel inverted pendulum testbed shown in Fig. 4. This platform is designed and developed at LIRMM ${ }^{6}$. Mechanical stops constrain the movement of the pendulum angle $\theta_{1}$.

6. LIRMM (Montpellier Laboratory of Informatics, Robotics and Microelectronics) : http ://www.lirmm.fr 


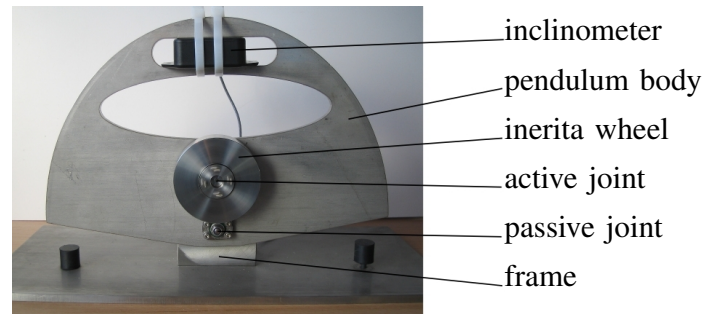

Fig. 4: The inertia wheel inverted pendulum

This angle is measured by an encoder linked to the actuator of the system (Maxon EC-powermax 30 DC motor). An inclinometer FAS-G of micro strain served to measure in real time the angle of the pendulum with respect to the vertical. The control approach is implemented on a computer using C++ language. The whole system is running under Ardence RTX real-time OS. Different control schemes have been developed and implemented on this testbed (Fig. 5) [21], [22], [23].

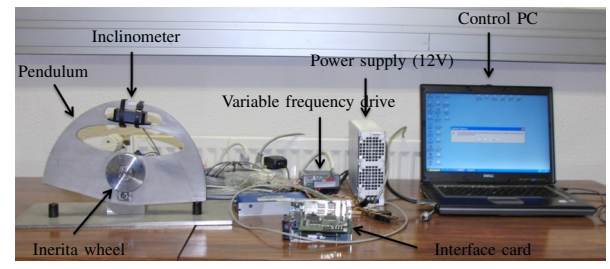

Fig. 5: IWIP experimental testbed

2) Stabilization with external disturbances: The two cases about external, matched and unmatched, disturbances are emphasized experimentally by two scenarios.

Scenario 1 : It is illustrated in Fig. 6. The external torques added to (19) are generated by pushing the pendulum at approximately $t=6 \mathrm{~s}, t=8 \mathrm{~s}$ and $t=12 \mathrm{~s}$. Experimental results are displayed in Fig. 7. The effect of punctual disturbances at different times can be observed as peaks on the curves. Compensations of such disturbances are observed in the evolution of the angular position and velocity. Matched disturbances are also compensated by the controller. We observe that the IDA-PBC controller compensates the added external torques and maintain the system around the desired equilibrium point.

Scenario 2 : The second type of external disturbances is illustrated in Fig. 8. It consists of an additional mass attached to the body of the pendulum. This mass generates a persistent torque applied to the pendulum (passive joint). Fig. 9 shows the obtained experimental results for the second scenario.

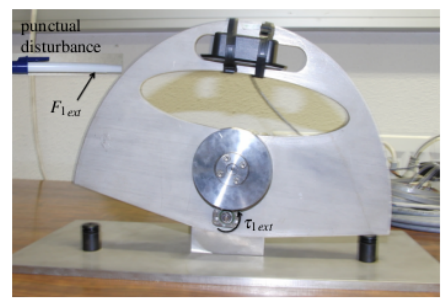

Fig. 6: Punctual disturbances applied to the pendulum
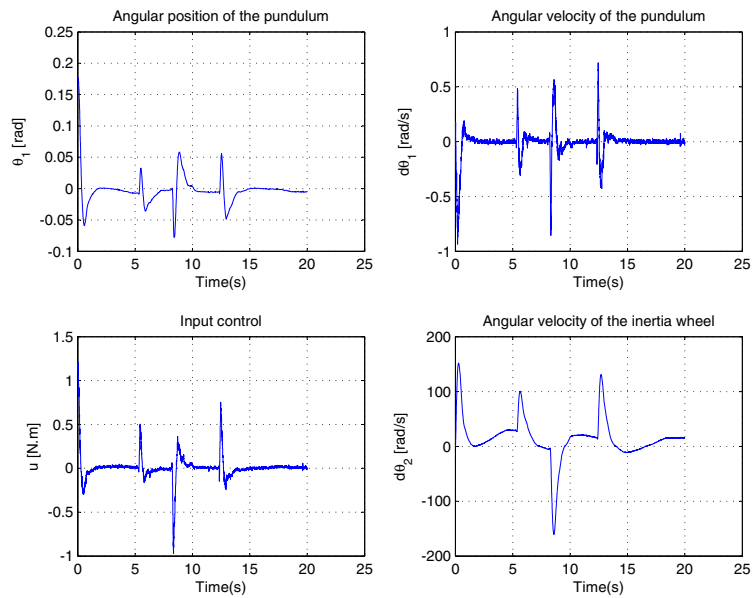

Fig. 7: Scenario 1 : Experimental results with external disturbances

Smooth stabilization is always guaranteed in spite of the existence of a persistent disturbance. The permanent rotation of the inertia wheel $\left(\dot{\theta}_{2}\right)$ permit to compensate the unmatched disturbances. As a result the IDA-PBC controller reject this type of disturbances and the pendulum is kept around its unstable equilibrium point.

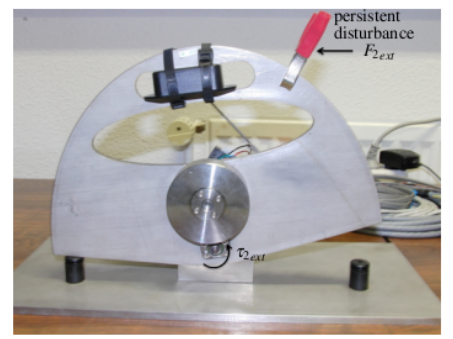

Fig. 8: Persistent disturbances applied to the pendulum 

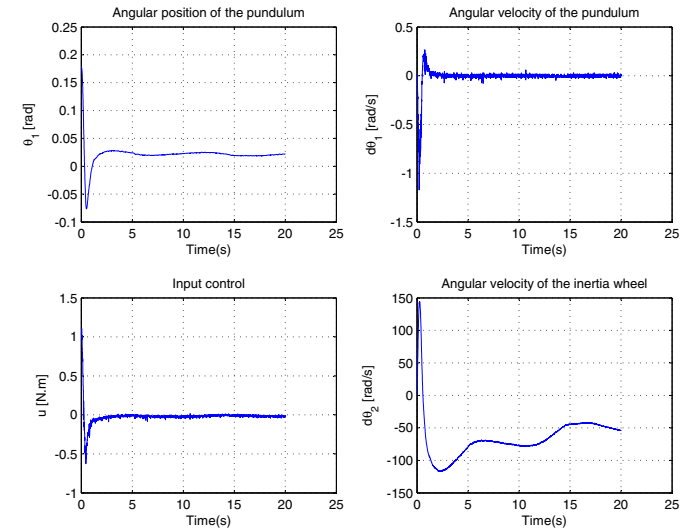

Fig. 9: Scenario 2 : Experimental results with external disturbances

\section{CONCLUSION AND FUTURE WORK}

This paper characterized the robustness of IDA-PBC control applied to a class of underactuated mechanical systems. The effect of external disturbances is especially studied in this paper. Two sufficient stability conditions are provided to deal with matched and unmatched disturbances. Motivated by the practical matter of IDA-PBC, this paper presented experimental results shown that IDA-PBC is robust with respect to external disturbances. The experimental platform used is an inertia wheel inverted pendulum developed at LIRMM. Based on results presented in this paper we note that in the presence of unmatched disturbances the controller cannot compensate static error. Extension of this work can be done in the sens of improving the robustness of IDA-PBC controller for a class of underactuated systems. An adaptive control can be proposed to compensate errors that are caused by uncertain parameters and unmatched disturbances.

\section{REFERENCES}

[1] F. Gomez-Estern, R. Ortega and M.W. Spong, "Total Energy Shaping for Underactuated Me-chanical Systems", 5th IFAC Symp. Nonlinear Control Systems, NOLCOS'01, St Petersburg, Russia, July 4-6, 2001.

[2] B. Maschke and A. van der Schaft. "Systems and networks : Mathematical theory and applications", volume II, pages 349-352. AcademicVerlag, 1994.

[3] A. D. Cerezo , PhD thesis "Modeling, simulation and control of a doubly-fed induction machine controlled by a back-to-back converter", Université Polytechnique de Catalunya, 2006.

[4] A. Van. Der. Schaft, "Port-Hamiltonian systems : an introductory survey", Proceedings of the International Congress of Mathematicians, Madrid, Spain, (2006) European Mathematical Society.
[5] R. Ortega, A. van der Schaft, B. Maschke and G. Escobar, "Interconnection and damping assignment passivity-based control of portcontrolled Hamiltonian systems", Automatica 38 (2002) 585-596.

[6] R. Ortega, M. Spong, F. Gomez-Estern, and G. Blankenstein, "Stabilization of a class of underactuated mechanical systems via interconnection and damping assignment", IEEE Transactions on Automatic Control, vol. 48, no. 8, pp. 1218-1233,2002.

[7] J.A. Acosta, R. Ortega, and A. Astolfi. "Interconnection and damping assignment passivity-based control of mechanical systems with underactuation degree one". In American Control Conference, 2004.

[8] H. Rodriguez, R. Ortega, and I. Mareels. "A novel passivity-based controller for an active magnetic bearing benchmark experiment". In Proc. Of the American Control Conference, 2000.

[9] H. Rodriguez, H. Siguerdidjane, and R. Ortega. "Experimental comparison of linear and nonlinear controllers for a magnetic suspension". InIEEE Proc. Conference on Control Applications, 2000.

[10] R. Ortega, A. Astolfi, G. Bastin, and H. Rodriguez. "Stabilization of food-chain systems using a port-controlled hamiltonian systems". In Proc. of the American Control Conference, 2000.

[11] V. Petrovic, R. Ortega, and A. Stankovic. "Interconnection and damping assignment approach to control of permanent magnet synchronous motor". IEEE Trans. Control Systems Technology, 9(6): :811-820, 2001.

[12] C. Batlle, A. Doria-Cerezo, and R. Ortega. "Power Flow Control of a Doubly-Fed Induction Machine Coupled to a Flywheel". European Journal of Control, 11(3):209-221, 2005.

[13] H. Rodriguez, R. Ortega, G. Escobar, and N. Barabanov. "A robustly stable output feedback saturated controller for the boost dc-to-dc converter". Systems and Control Letters, $40: 1-8,2000$.

[14] R. Ortega and E. G. Canseco, "Interconnection and Damping Assignment Passivity-Based Control : A Survey", European Journal of Control (2004) $10: 432-450$.

[15] L. Gentili and A.J. van der Schaft. "Regulation and input disturbance suppression for port-controlled hamiltonian systems". InProc. 2nd IFAC Workshop on Lagrangian and Hamiltonian Methods for Nonlinear Control, 2003.

[16] C. Batlle, A. Doria-Cerezo, and E. Fossas. "Improving the robustness of Hamiltonian passive control". Technical report, IOC, Technical University of Catalonia, 2005.

[17] M. Bechrif, E. Mendes, "Stability and robustness of disturbed-port controlled Hamiltonian systems with dissipation", Proceedings of the 16th World Congress of the International Federation of Automatic Control, Th-A03-TO/3 16th IFAC World Congress Prague (2005).

[18] J. G. Romero, A. Donaire, R. Ortega, "Robust energy shaping control of mechanical systems", Systems and Control Letters 62(2013) 770-780, Elsevier.

[19] M. Spong and M. Vidyasagar, "Robot Dynamics and Control"“. New York, USA : John Wiley and Sons, 1989.

[20] V. Santibañez, R. Kelly and J. Sandoval, "Control of the Inertia Wheel Pendulum by Bounded Torques", 44th Conference on Decision and Control, and the European Control Conference 2005, pp : 8266-8270.

[21] S. Andary, A. Chemori, and S. Krut, "Control of the Underactuated Inertia Wheel Inverted Pendulum for Stable Limit Cycle Generation", RSJ Advanced Robotics, vol. 23, no. 15, pp. 1999-2014, 122009.

[22] S. Andary, A. Chemori, and S. Krut, "Estimation-based disturbance rejection in control for limit cycle generation on inertia wheel inverted pendulum testbed", IROS'09 : Proceedings of the 2009 IEEE/RSJ international conference on Intelligent robots and systems. Piscataway, NJ, USA : IEEE Press, 2009, pp. 1302-1307.

[23] A. Chemori, S. Krut, N. Touati, "Le pendule inversé stabilisé par volant d'inertie, un système non linéaire sous-actionné", 3èmes Journées Démonstrateurs 2010, Angers : France (2010). 